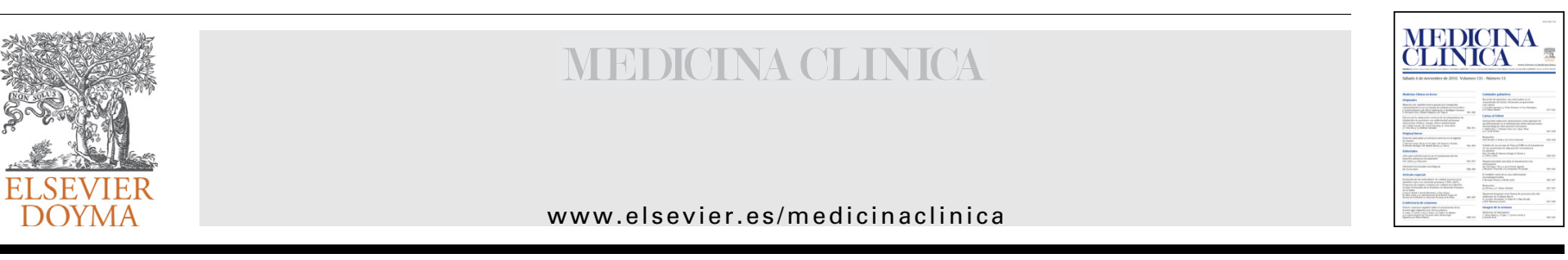

Original breve

\title{
Efecto de masticar chicle sobre la halitosis
}

\section{Fiorella De Luca-Monasterios *, Eduardo Chimenos-Küstner y José López-López}

Departamento de Odontoestomatología, Facultad de Odontología, Campus Universitario de Bellvitge, Universidad de Barcelona, L'Hospitalet de Llobregat, Barcelona, España

I N F O R M A C I Ó N D E L A R T ÍC U L O

Historia del artículo:

Recibido el 22 de septiembre de 2013

Aceptado el 28 de noviembre de 2013

On-line el $\mathrm{xxx}$

\section{Palabras clave:}

Halitosis

Monitor de sulfuros

Compuestos volátiles sulfurados

Prueba organoléptica

Chicle

\begin{abstract}
R E S U M E N
Fundamento y objetivo: Este estudio pretende estimar la prevalencia de halitosis oral en una población joven y mostrar el efecto del chicle sobre el aliento.

Pacientes y método: Estudio prospectivo de campo descriptivo correlacional, de corte transversal. Se seleccionaron a conveniencia 121 jóvenes, de los que 98 concluyeron el estudio, el cual se realizó en el Hospital Odontológico Universitario-Universidad de Barcelona. El protocolo consistió en: cuestionario, evaluación clínica oral, prueba organoléptica (PO) y medición de compuestos volátiles sulfurados (CVS) con monitor de sulfuros, antes y después de masticar chicle durante $15 \mathrm{~min}$, con 2 investigadores calibrados.

Resultados: Un $87,8 \%$ tenían higiene oral adecuada, el 17,3\% refirieron mal aliento, y un 29,6\%, xerostomía. En la PO 44 sujetos tenían un grado $\geq 2$; la concordancia de examinadores fue del $75 \%$. La determinación de CVS en 53 individuos fue $\leq 100$ partes por billón (ppb) y en 45 sujetos $>100 \mathrm{ppb}$. Se obtuvo una reducción de CVS post de 17,34\%, con una media de disminución de 1,8-9,0 ppb ( $\mathrm{p}=0,003$ ). Conclusiones: La prevalencia de halitosis fue del $36,7 \%$. El uso de chicle como adyuvante en casos de halitosis disminuye los CVS, mejorando la percepción de terceros y del propio paciente.
\end{abstract}

(c) 2013 Elsevier España, S.L. Todos los derechos reservados.

\section{Effect of chewing gum on halitosis}

\begin{abstract}
A B S T R A C T
Background and objective: This study aims to estimate the prevalence of oral halitosis in a young population and show the effect of chewing gum on their breath.

Patients and method: Prospective, descriptive correlational cross section study. We selected a convenience sample of 121 young individuals and 98 completed the study. It was carried out at the University Dental Hospital-University of Barcelona. The protocol consisted of: questionnaire, oral clinical evaluation, organoleptic tests (OT) and measurement of volatile sulfur compounds (VSC) with sulphide monitoring before and after chewing gum during $15 \mathrm{~min}$, with 2 calibrated investigators. Results: A percentage of 87.8 had adequate oral hygiene, $17.3 \%$ reported bad breath and $29.6 \%$ had xerostomia. Forty-four subjects had a OT grade $\geq 2$; the agreement of examiners was $75 \%$. The VSC in 53 individuals were $\leq 100$ parts per billion (ppb) and in 45 subjects, it was $>100 \mathrm{ppb}$. A post-reduction of VSC of $17.34 \%$ was obtained with a mean decrease of $1.8-9.0 \mathrm{ppb}(P=.003)$.

Conclusions: The prevalence of halitosis was $36.7 \%$. The use of chewing gum as an adjunct in cases of halitosis decreases the VSC, improving the perception of others and the patient.
\end{abstract}

(c) 2013 Elsevier España, S.L. All rights reserved.

\section{Introducción}

La halitosis es un olor desagradable del aire bucal exhalado, queja frecuente en el odontólogo y médico generalista ${ }^{1-3}$. Las causas son múltiples, principalmente intraorales, asociadas al mal

\footnotetext{
* Autor para correspondencia.

Correo electrónico: fiorella.dlm@gmail.com (F. De Luca-Monasterios).
}

estado oral: enfermedad periodontal, saburra lingual, caries y sequedad bucal $^{1,3}$, y además, la degradación por diferentes bacterias del material orgánico presente en la boca produce sustancias aromáticas, especialmente compuestos volátiles sulfurados $(\mathrm{CVS})^{1}$. Para medir el aliento se recurre a entrevistas sobre autopercepción (AP), pruebas organolépticas (PO) realizadas por examinadores entrenados y valoración objetiva mediante aparatos de medición, como monitores de sulfuros y de cromatografía de gases, que expresan resultados en partes por billón (ppb). Suele

0025-7753/\$ - see front matter ๔ 2013 Elsevier España, S.L. Todos los derechos reservados.

http://dx.doi.org/10.1016/j.medcli.2013.11.038 
descartarse el mal aliento con valores de CVS $<100 \mathrm{ppb}^{3}$, otros hablan de halitosis con valores $\geq 75 \mathrm{ppb}^{1}$.

Una boca sana y limpia es la mejor medida para tratar el problema. El uso de chicles y/o colutorios con antimicrobianos disminuye la puntuación de $\mathrm{PO}^{1,4,5}$.

El objetivo del estudio es evaluar la prevalencia de halitosis en una población joven y determinar el efecto que produce el chicle sobre el aliento antes y después de masticarlo $15 \mathrm{~min}$.

\section{Pacientes y método}

Se realiza un muestreo de conveniencia en estudiantes de Odontología. Los sujetos seleccionados completaron un cuestionario sobre mal aliento (tabla 1). Las visitas se realizaron en el Hospital Odontológico de la Universidad Barcelona.

Criterios de inclusión: estudiantes voluntarios con o sin halitosis. Criterios de exclusión: sujetos con enfermedades sistémicas controladas (diabetes mellitus, trastornos hepáticos, renales o gástricos, e infección por el virus de la inmunodeficiencia humanasida), embarazadas, sujetos medicados en las últimas 4 semanas con antibióticos o medicamentos xerostomizantes o alérgicos a componentes del chicle, y pacientes portadores de prótesis.

\section{Métodos}

Los 121 individuos elegidos debían evitar en las $48 \mathrm{~h}$ previas alimentos odoríferos, suprimir el consumo de bebidas alcohólicas, carbonatadas o café, aperitivos, dulces, mascar chicle, fumar, cepillarse o enjuagarse con colutorio una hora antes del estudio. Las mediciones se realizaron por la mañana por 2 examinadores, antes y después de la masticación de chicle. Parámetros clínicos: se evaluaron, mediante exploración oral, las alteraciones bucales susceptibles de causar halitosis (caries, enfermedad periodontal y mucosa, portadores de aparatología-prótesis fija o removible, sequedad, etc.). Para valorar el nivel de higiene oral se usó el índice de Greene y Vermillion (IHO-s) 6 . La PO fue determinada por 2 exploradores calibrados, mediante la escala de Rosenberg ${ }^{2,7}$ (valora de 0 a 5). De no coincidir la puntuación entre los examinadores, se tomó el valor más alto. Los CVS se registraron cuantitativamente en $\mathrm{ppb}$ con un monitor de sulfuros (modelo RH17E, Halimeter $\AA$, Interscan Corporation, Chatsworth, CA, EE. UU.). Se realizaron 3 lecturas en cada sujeto, tanto prechicle como poschicle, tomándose el valor medio. Se considera halitosis con $\geq 75$ ppb y PO positiva $\geq 2$. La medición del pH lingual con tiras reactivas de papel (Indicador Universal $\mathrm{pH} 1-11$, Panreac Química S. A., Barcelona, España) se hizo en la región posterior del dorso lingual. El chicle masticado consistía en grageas de 1,4 g (Orbit ${ }^{\circledR}$ menta de Wrigley Co., S. L. U., Barcelona, España), del cual se hacen 60 ciclos/min por $15 \mathrm{~min}$.

\section{Análisis estadístico}

El análisis estadístico se realizó mediante el software SPSS ${ }^{\mathbb{R}}$ Statistics de IBM ${ }^{\circledR}$ para Windows ${ }^{\circledR}$, versión 20.0 (IBM Corp 2011, Armonk, NY, EE. UU.). Se efectuó un análisis descriptivo convencional mediante tablas de frecuencia para las variables cualitativas, medias y desviaciones cuantitativas. Para la comparación de medias entre grupos independientes se empleó el t-test, con la corrección de Welch en las variancias heterogéneas; si era preciso se empleó el test no paramétrico de Mann-Whitney. Para la independencia entre variables cualitativas se empleó el test razón de verosimilitud (G-test), y el test exacto de Fisher cuando el número esperado era $<5$. La asociación entre variables cuantitativas se evaluó mediante la prueba de correlación de rangos de Spearman, ya que las variables estudiadas presentan distribuciones marcadamente asimétricas. Para la comparación de medias con datos emparejados se empleó el t-test o la alternativa no paramétrica de la $\mathrm{W}$ de Wilcoxon, mientras que en variables cualitativas se empleó el test de McNemar.

\section{Resultados}

De 121 individuos seleccionados, 18 abandonaron por falta de tiempo y 2 por no seguir las instrucciones de las pruebas. Quedaron para el análisis 98 sujetos, 66,3\% eran mujeres y 33,7\% varones, con un intervalo de edad de 18 a 40 años (media de 24,7; intervalo de confianza del 95\% [IC 95\%] 23,6-25,8 años), no habiéndose observado diferencia significativa por sexo o edad $\left(t-\right.$ test $_{\mathrm{w}}=1,76$; $\mathrm{gl}=46,4 ; \mathrm{p}=0,009$ ) (tabla 2 ).

Un $27,6 \%$ de los pacientes fumaban cigarrillos (9 varones y 18 mujeres), con una media de 8 cigarrillos/día, y el 26,5\% ingerían alcohol ocasionalmente. Un $5,1 \%$ señaló ser respirador bucal diurno, y un $8,16 \%$, nocturno. La mayoría de los pacientes $(77,55 \%)$ expresó cepillar sus dientes entre 2 y 3 veces/día. Utilizaban

Tabla 1

Cuestionario corto de salud

1. ¿Siente sequedad bucal en este momento? Sí

2. ¿Ha tenido mal aliento (halitosis) en alguna ocasión? Sí___ No

3. ¿Siente mal aliento en este momento? Sí ___ No

4. ¿Cuándo ha sentido mal aliento?

5. ¿En qué momento del día comúnmente ha presentado mal aliento? Mañana _ Tarde _ Noche _ Todo el día_

6. ¿Con qué frecuencia suele tener halitosis? Nunca _ Casi nunca_ A veces _ A menudo__

7. ¿A qué le atribuye su causa? Alimento __, mala higiene oral _ ¿Cuál?

8. ¿Cómo supo que tenía mal aliento? Comunicación no verbal de personas _ _ Alguien se lo dijo __ ¿Quién? ver que las personas alrededor actúan diferentes _, otro

9. ¿Alguna vez ha pasado por una de estas circunstancias a causa de su mal aliento?: Evitar hablar con otras personas_ Timidez cuando alguien se le acerca _ No le agrada conocer a otras personas__ No puede iniciar una relación _ Otras personas le evitan__ Otras: estos

10. ¿Su halitosis afecta a su vida? No Sí ¿De qué manera?

11. ¿Sufre de estrés o ansiedad de manera personal o profesional? Sí, mucho-siempre _ Sí, a veces-poco __ No, nunca_

12. ¿Comúnmente presenta resfriado o alergias? No_Sí__Con qué frecuencia?

13. ¿Tiene que limpiar su nariz constantemente? No__ Sí__ ¿Con qué frecuencia?

14. ¿Qué ha utilizado para combatir su mal aliento? Nada_, Enjuague bucal _, Spray _ Chicle _, Dulces__, Evita ciertos alimentos, a saber: Otros

15. ¿Ha consultado a cualquier otro médico a causa de su halitosis? No_ Sí__ ¿A cuál? Dentista _ Médico general _ Otorrino _ Internista__ Otro ¿Cuándo?

16. ¿Ha sufrido de algunas de las siguientes afecciones? Sinusitis _ Enfermedades de la nariz _ Respira por la boca _ Ronca _ Problemas estomacales_ Reflujo gastroesofágico _ Enfermedad pulmonar o bronquitis _ Enfermedades del hígado _ Sequedad bucal _ Dolor de garganta-amígdalas _ Infección bacteriana _ Obstrucción intestinal-estreñimiento _ Condiciones psíquicas _ Otros 
Tabla 2

Datos demográficos, anamnesis y exploración clínica inicial

\begin{tabular}{|c|c|c|}
\hline Datos & $\mathrm{n}$ & Frecuencia \\
\hline \multicolumn{3}{|l|}{ Edad (años) } \\
\hline 20 a 22 & 49 & 50,0 \\
\hline 23 a 25 & 25 & 25,5 \\
\hline 26 a 40 & 24 & 24,5 \\
\hline \multicolumn{3}{|l|}{ Sexo } \\
\hline Mujer & 65 & 66,3 \\
\hline Varón & 33 & 33,7 \\
\hline \multicolumn{3}{|l|}{ Enfermedad actual } \\
\hline No & 79 & 80,6 \\
\hline Sí & 19 & 19,4 \\
\hline \multicolumn{3}{|l|}{ Medicación } \\
\hline No & 78 & 79,6 \\
\hline Sí & 20 & 20,4 \\
\hline \multicolumn{3}{|l|}{ Fumador } \\
\hline No & 71 & 72,4 \\
\hline Sí & 27 & 27,6 \\
\hline \multicolumn{3}{|c|}{ Autopercepción halitosis } \\
\hline No & 81 & 82,7 \\
\hline Sí & 17 & 17,3 \\
\hline \multicolumn{3}{|c|}{ Autopercepción sequedad bucal } \\
\hline No & 69 & 70,4 \\
\hline Sí & 29 & 29,6 \\
\hline \multicolumn{3}{|l|}{ Condición lengua } \\
\hline Normal & 72 & 73,5 \\
\hline Saburral & 15 & 15,3 \\
\hline Seudopatológica & 11 & 11,2 \\
\hline \multicolumn{3}{|l|}{ Enfermedad oral } \\
\hline No & 88 & 89,8 \\
\hline Sí & 10 & 10,2 \\
\hline \multicolumn{3}{|l|}{ Higiene bucal } \\
\hline Excelente & 37 & 37,8 \\
\hline Buena & 49 & 50,0 \\
\hline Regular & 6 & 6,1 \\
\hline Deficiente & 6 & 6,1 \\
\hline \multicolumn{3}{|l|}{ pH lingual (pre) } \\
\hline$\leq 6,9$ & 40 & 40,8 \\
\hline$\geq 7$ & 58 & 59,2 \\
\hline Total $\mathrm{n}$ & 98 & \\
\hline
\end{tabular}

enjuague bucal e hilo dental al menos una vez/día (18,36 y 7,14\%, respectivamente). Los pacientes atribuían la intensificación de la halitosis a alimentos olorosos (53,06\%), higiene oral (36,7\%), trastornos digestivos $(7,14 \%)$ y causas nasales $(5,10 \%)$.

El $17,3 \%$ refirió sentir halitosis por AP y $29,6 \%$ señaló xerostomía. Solo 19 sujetos refirieron alguna enfermedad actual: asma (3,06\%), psoriasis $(2,04 \%)$ y afecciones del tracto respiratorio superior y garganta (11,22\%). En la consulta inicial, el $20,4 \%$ confirmó ingesta de fármacos (anticonceptivos orales, descongestionantes, ansiolíticos, antibióticos y antihistamínicos). Los hallazgos de la exploración oral fueron lengua saburral $(15,3 \%)$ y enfermedad periodontal y dental (10,2\%) (tabla 2 ).

En general, los sujetos presentaron una higiene oral adecuada, con una media de IHO-s $=0,44$ (IC 95\% 0,27-0,61). Un 37,8\% presentó una buena higiene, y en el $50 \%$ era excelente. Los sujetos con AP de halitosis también gozaban de buena higiene (media $=0,35$ ), sin significación con respecto a los que no tenían AP. Se detectó asociación significativa entre IHO-s y PO (U$\mathrm{MW}=1.733 ; \mathrm{p}<0,001)$, con un IHO-s más bajo en individuos con PO no perceptible. De modo similar, los valores halitométricos presentaron correlación positiva moderada, estadísticamente significativa, IHO-s (Spearman $r=0,34 ; n=98 ; p=0,001$ ): a mayor IHOS-s más CVS. Por el contrario, la higiene bucal no presentaba diferencias significativas atendiendo a la AP de halitosis (UMW $=672 ; \mathrm{p}=0,87$ )

En la tabla 3 se resumen los resultados a partir de las pruebas realizadas en la primera fase del estudio. Agrupamos la escala organoléptica en 2 categorías, considerando valores de 0 y 1 como olor "no perceptible» y de 2 a 5 olor "perceptible». Del total de participantes, 17 afirmaron AP de halitosis. Al comparar estos resultados con la $\mathrm{PO}, 8$ arrojaron un resultado de "sin olor», mientras que en 9 fue perceptible. Los 81 sujetos restantes no refirieron halitosis; sin embargo, los exploradores la detectaron en 35 de ellos, no observándose asociación significativa entre estas variables (G-test $=0,53 ; \mathrm{p}=0,46$ ) (tabla 3 ). La valoración de PO realizada por los evaluadores tuvo un buen nivel de concordancia mediante el test kappa, siendo de 0,75 para el análisis pre y de 0,72 para el post masticar chicle. Al comparar los resultados de las concentraciones medias de CVS entre los 2 grupos definidos por AP, no se encontraron diferencias significativas (U-MW $=727$; $\mathrm{p}=0,72$ ). Los individuos sin AP tenían lecturas de CVS más bajas (109 ppb) que los que afirmaron AP de halitosis (140 ppb), con una desviación típica entre 85,4 y 140,2 , respectivamente (tabla 3). La distribución de CVS fue en 53 sujetos $\leq 100 \mathrm{ppb}$, y en 45 sujetos $>100 \mathrm{ppb}$, con una media global de $91 \mathrm{ppb}$.

Por otra parte, no existió asociación significativa entre fumar y la AP de halitosis (G-test $=1,80 ; \mathrm{p}=0,18$ ), ni con la PO (Gtest $=0,03 ; p=0,98)$. Tampoco se encontró diferencia significativa con los valores medios de CVS (U-MW $=782 ; \mathrm{p}=0,16$ ), siendo de $93,8 \mathrm{ppb}$ en individuos fumadores y de $123,1 \mathrm{ppb}$ en no fumadores. Del total, 36 individuos (36,7\%) resultaron positivos tanto en la PO $(\geq 2)$ como en la halitometría ( $\geq 75 \mathrm{ppb}$ ), considerados con halitosis.

La PO después de la masticación indicó que en 21 sujetos persistía. Si comparamos los valores de PO con los CVS tanto pre como post, se evidenció que ante PO perceptibles, los CVS eran más altos. En varones, la media pre fue de $117,3 \mathrm{ppb}$ y la de post de $106,5 \mathrm{ppb}$; en mujeres estos valores fueron de $113,9 \mathrm{ppb}$ para pre y de $103,8 \mathrm{ppb}$ para post (tabla 3 ). Por otra parte, 17 individuos obtuvieron una mejoría tras la $\mathrm{PO}$, mientras que ninguno empeoró, lo que supone un incremento significativo de individuos con olor no detectable $($ McNemar $=15,1 ; \mathrm{p}<0,001)$. Del mismo modo, en la halitometría la disminución de CVS fue significativa poschicle (Wilcoxon $=1.590 ; \mathrm{p}=0,003$ ), aunque de limitada magnitud (IC 95\% 1,8-9,0 ppb). El pH del dorso lingual no fue significativo entre ambas evaluaciones (Wilcoxon $=1.295 \mathrm{y} \mathrm{p}=0,57$ ) (tabla 3 ).

\section{Discusión}

A diferencia de otros autores ${ }^{2,8}$, no encontramos desigualdades por sexo. En el presente estudio el nivel de caries fue muy bajo, solo

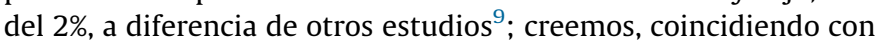
Arinola y Olukoju ${ }^{10}$, que algunas de las razones pueden ser la edad, el nivel educativo y socioeconómico de la población estudiada y el carácter/actitud del voluntario.

Diferentes estudios postulan que el uso del chicle puede afectar positivamente la condición de la lengua saburral, debido a la función mecánica y química que esta ejerce al masticar ${ }^{11}$. En el presente trabajo, al igual que en otros ${ }^{12}$, no se encontraron diferencias significativas en cuanto al $\mathrm{pH}$ del dorso lingual.

En este estudio se priorizaron las PO, consideradas como el estándar de oro para la evaluación del mal aliento ${ }^{4}$, resultando una buena correlación entre examinadores (kappa $=0,75$ ). La media de PO pre fue de 2,4, 4 décimas superior a la del estudio de Keller et al. ${ }^{4}$. Nuestros resultados revelan que 44 individuos tenían una PO inicial $\geq 2$, y en un $56,1 \%$ la AP inicial se correlaciona comparativamente con las PO, logrando, además, una disminución de los CVS (1,8-9,0 ppb) poschicle, siendo significativo (Wilcoxon $=1.590 ; p=0,003)$, de manera semejante a las que se 
Tabla 3

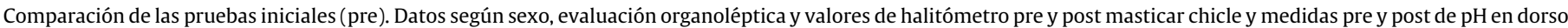
lingual, evaluación organoléptica y medición de compuestos volátiles sulfurados

\begin{tabular}{|c|c|c|c|c|c|c|c|c|c|}
\hline \multicolumn{3}{|c|}{ Autopercepción halitosis } & \multicolumn{4}{|c|}{ Evaluación organoléptica } & \multicolumn{3}{|c|}{ Halitometrías (CVS en ppb) } \\
\hline \multirow{3}{*}{$\begin{array}{l}\text { Respuesta } \\
\text { No } \\
\text { Sí }\end{array}$} & \multirow{3}{*}{$\begin{array}{l}\mathrm{n} \\
81 \\
17\end{array}$} & \multirow{2}{*}{$\begin{array}{l}\text { Frecuencia } \\
82,70\end{array}$} & & Olor no perceptible & \multicolumn{2}{|c|}{ Olor perceptible } & \multirow{3}{*}{$\begin{array}{l}\text { Media } \\
109,0 \\
140,5\end{array}$} & \multirow{3}{*}{$\begin{array}{l}\text { Mediana } \\
90,0 \\
98,0\end{array}$} & \multirow{2}{*}{$\begin{array}{l}\text { DT } \\
85,4\end{array}$} \\
\hline & & & 46 & $56,8 \%$ & 35 & $43,2 \%$ & & & \\
\hline & & 17,30 & 8 & $47,1 \%$ & 9 & $52,9 \%$ & & & 140,2 \\
\hline \multicolumn{3}{|l|}{ Datos } & \multicolumn{2}{|c|}{ Valores pre } & & \multicolumn{4}{|c|}{ Valores post } \\
\hline \multirow{2}{*}{\multicolumn{2}{|c|}{ Evaluación organoléptica }} & \multicolumn{4}{|c|}{ CVS (ppb) } & \multicolumn{4}{|c|}{ CVS (ppb) } \\
\hline & & $\mathrm{n}$ & Frecuencia & Media & DT & $\mathrm{n}$ & Frecuencia & Media & $\overline{\text { DT }}$ \\
\hline \multicolumn{10}{|l|}{ Sexo } \\
\hline \multicolumn{10}{|l|}{ Varón } \\
\hline Olor no & & 20 & 60,6 & 82,7 & 28,8 & 26 & 78,8 & 84,3 & 34,8 \\
\hline Olor pe & & 13 & 39,4 & 170,5 & 138,9 & 7 & 21,2 & 189,3 & 90,9 \\
\hline \multicolumn{10}{|c|}{ Mujer } \\
\hline Olor no & & 34 & 52,3 & 75,7 & 37,7 & 45 & 69,2 & 84,8 & 51,5 \\
\hline Olor pe & & 31 & 47,7 & 155,8 & 123,0 & 20 & 30,8 & 146,5 & 96,2 \\
\hline Total & & 98 & & & & & & & \\
\hline \multirow{2}{*}{\multicolumn{2}{|c|}{ Datos }} & & \multicolumn{3}{|c|}{ Valores pre } & & \multicolumn{3}{|c|}{ Valores post } \\
\hline & & & $\mathrm{n}$ & & Frecuencia & & $\mathrm{n}$ & & Frecuencia \\
\hline \multicolumn{10}{|l|}{ pH lingual } \\
\hline$<7$ & & & 40 & & 40,8 & & 27 & & 27,6 \\
\hline$=7$ & & & 20 & & 20,4 & & 42 & & 42,9 \\
\hline$>7$ & & & 38 & & 38,8 & & 29 & & 29,6 \\
\hline \multicolumn{10}{|c|}{ Evaluación organoléptica } \\
\hline Olor no $\mathrm{p}$ & & & 54 & & 55,1 & & 71 & & 72,4 \\
\hline Olor perc & & & 44 & & 44,9 & & 27 & & 27,6 \\
\hline \multicolumn{10}{|l|}{ CVS (ppb) } \\
\hline Valor $\leq 6$ & & & 26 & & 26,5 & & 30 & & 30,6 \\
\hline $65<$ valor & & & 27 & & 27,6 & & 29 & & 29,6 \\
\hline $100<$ val & & & 21 & & 21,4 & & 16 & & 16,3 \\
\hline $125<$ valc & & & 24 & & 24,5 & & 23 & & 23,5 \\
\hline
\end{tabular}

CVS: compuestos volátiles sulfurados; DT: desviación típica; ppb: partes por billón.

consiguieron con chicle de eucalipto $(p=0,034)^{11}$. Los valores pre según sexo fueron: PO significativamente superior en varones que en mujeres (82-170 ppb y 75-155 ppb, respectivamente), así como los valores post (media en varones $189 \mathrm{ppb}$ y en mujeres $146 \mathrm{ppb}$, concordando con otras investigaciones: $161 \mathrm{ppb}$ en varones y $141 \mathrm{ppb}$ en mujeres) $)^{2}$. Sin embargo, otros autores consideran halitosis cuando los CVS son $\geq 110 \mathrm{ppb}^{13}$. La media de todos los CVS registrados en este estudio con PO positiva estuvo dentro de lo esperado (141 ppb), en comparación con la obtenida en otros estudios en pacientes con halitosis ${ }^{2,8}$. La disminución de la halitosis tras masticar chicle durante $15 \mathrm{~min}$ se confirmó por reducción del mal olor bucal en las PO y los CVS medidos.

Coincidiendo con otros trabajos ${ }^{4,11}$, obtuvimos que el uso del chicle contribuye a la reducción y prevención del mal olor bucal en la población joven, cuando se parte de valores de halitosis bajos y moderados.

Este estudio presentó limitaciones en cuanto a la selección de los participantes, ya que el muestreo fue de conveniencia. Por otra parte, los factores que influyen (incluidos en el cálculo de cada variable dependiente) fueron los datos subjetivos del cuestionario y la anamnesis, y los hallazgos objetivos de la exploración clínica, lo que podría representar cierto sesgo en la información. Consideramos necesario realizar nuevos estudios que instauren métodos de diagnóstico y tratamientos más fiables y concluyentes.

\section{Financiación}

El estudio se ha soportado parcialmente mediante una ayuda a la investigación de estudiantes de posgrado (XIII convocatoria de la Facultad de Odontología-Universidad de Barcelona).

\section{Conflicto de intereses}

Los autores declaran no tener ningún conflicto de intereses.

\section{Bibliografía}

1. Rösing CK, Loesche W. Halitosis: An overview of epidemiology, etiology and clinical management. Braz Oral Res. 2011;25:466-71.

2. Quirynen M, Dadamio J, van den Velde S, de Smit M, Dekeyser C, van Tornout M, et al. Characteristics of 2000 patients who visited a halitosis clinic. J Clin Periodontol. 2009;36:970-5.

3. Bollen CM, Beikler T. Halitosis: The multidisciplinary approach. Int J Oral Sci. 2012;4:55-63.

4. Keller MK, Bardow A, Jensdottir T, Lykkeaa J, Twetman S. Effect of chewing gums containing the probiotic bacterium Lactobacillus reuteri on oral malodour. Acta Odontol Scand. 2012;70:246-50.

5. López-López J, Jané Salas E, Chimenos Küstner E. Prognosis and treatment of dry mouth. Systematic review. Med Clin (Barc). 2014;142:119-24.

6. Greene JC, Vermillion JR. The simplified oral hygiene index. J Am Dent Assoc. $1964 ; 68: 7-13$

7. Rosenberg M, Kulkarni GV, Bosy A, McCulloch CA. Reproducibility and sensitivity of oral malodor measurements with a portable sulphide monitor. J Dent Res. 1991;70:1436-40.

8. Seemann R, Bizhang M, Djamchidi C, Kage A, Nachnani S. The proportion of pseudo-halitosis patients in a multidisciplinary breath malodour consultation. Int Dent J. 2006:56:77-81.

9. Nalcaci R, Baran I. Oral malodor and removable complete dentures in the elderly. Oral Surg Oral Med Oral Pathol Oral Radiol Endod. 2008;105:e5-9.

10. Arinola JE, Olukoju OO. Halitosis amongst students in tertiary institutions in Lagos state. Afr Health Sci. 2012;12:473-8.

11. Tanaka M, Toe M, Nagata H, Ojima M, Kuboniwa M, Shimizu K, et al. Effect of eucalyptus-extract chewing gum on oral malodor: A double-masked, randomized trial. J Periodontol. 2010;81:1564-71.

12. Tolentino Ede S, Chinellato LE, Tarzia O. Saliva and tongue coating $\mathrm{pH}$ before and after use of mouthwashes and relationship with parameters of halitosis. J Appl Oral Sci. 2011;19:90-4.

13. Liu XN, Shinada K, Chen XC, Zhang BX, Yaegaki K, Kawaguchi Y. Oral malodorrelated parameters in the Chinese general population. J Clin Periodontol. 2006;33:31-6. 